\title{
EDITORIAL
}

\section{Overcoming a stigma: the lung cancer patient in the intensive care unit}

\author{
N. Schönfeld* and J-F. Timsit*,ף
}

B efore embarking on a discussion of the lung cancer patient in intensive care, it is important to clarify that this editorial will not be referring to the group of patients with a good prognosis having undergone surgery with a curative intent, but to patients whose malignancies more or less determine the outcome in terms of survival time, and who are suffering from acute or subacute deterioration in the form of a complication resulting either from the underlying disease or tumour or from treatment-related sequelae. In such cases, most doctors' knowledge and experience will be confounded with those dark emotions that serve to sustain a misconception that is still associated with lung cancer patients, despite certain advances in treatment achieved through multimodal regimes and "new drugs".

As the technical possibilities of intensive medicine, including in particular the improvement of ventilation techniques with varying degrees of invasiveness, increase, it seems desirable to take a fresh look at the survival and prognostic factors in lung cancer patients admitted to a medical intensive care unit (ICU). ADAM and SOUBANI [1] have taken up this challenge. Their results are reported in the present issue of the European Respiratory Journal. At first sight, the range of reasons leading to referral for intensive care appears quite familiar and unspectacular: first, pneumonia, followed by exacerbated chronic obstructive pulmonary disease, pulmonary oedema and haemoptysis. As with most studies of this kind [2, 3], the analysis is retrospective. However, that does not, by any means, detract from its evidential value, since the criteria on which referrals of critically ill patients with nonmalignant disease to intensive care have been based to date fail to do justice to lung cancer patients with their complex requirements $[4,5]$. As in other recent studies published in the field $[2,3,6]$, it was found that short-term mortality is mainly related to the severity of organ dysfunction and not to the characteristics of the malignancy [1].

\footnotetext{
*Lungenklinik Heckeshorn, HELIOS Klinikum Emil von Behring, Berlin, Germany. " INSERM U823, Outcome of cancers and critical illnesses, Albert Bonniot Institute Research Centre, La Tronche, and 'Medical Intensive Care Unit, A. Michallon University Hospital, Grenoble, France.

STATEMENT OF INTEREST: None declared

CORRESPONDENCE: N. Schönfeld, Lungenklinik Heckeshorn, HELIOS Klinikum Emil von Behring, Walterhöferstr. 11, 14165 Berlin, Germany. Fax: 49 3081022778. E-mail: Schoenfeld.Berlin@Tonline.de; J-F. Timsit, Medical Intensive Care Unit, University Hospital A. Michallon, BP 217, 38043 Grenoble Cedex 9, France. Fax: 33 476765569. E-mail: jftimsit@chu-grenoble.fr
}

Like other authors before them, ADAM and SOUBANI [1] confirm, once again, that the most complex task facing the ICU physician is deciding on the indication for and performing ventilation therapy in lung cancer patients. However, unlike older studies, the study by ADAM and SOUBANI [1] reveals an encouraging trend towards improved results for certain groups of patients, which can be attributed to three factors: 1) patients with poor functional status or patients with no lifeprolonging treatment may be denied admission to the ICU [7]; 2) earlier referral of cancer patients to the ICU for noninvasive diagnostic and therapeutic strategies, provided that these strategies do not delay intubation and optimal management [8]; and 3) a better knowledge of certain complications that develop in critically ill patients with malignancies.

What is more important and far exceeds anything that can be learnt from figures is that the authors finally come to the conclusion that to undergo intensive care, including mechanical ventilation, is by no means fruitless for lung cancer patients. However, while the authors rightly call for prospective studies, we must ask how, apart from employing algorithms based on the prognosis and other factors generally taken into account in clinical research on intensive care, future studies can be designed in such a way that they help to arrive at a sounder basis for making decisions. Put another way, why is the lung cancer patient an exceptional case?

In a more general way, where ICU care does not seem obviously futile, the intensivist may propose unlimited management for a limited period if patients and/or family are willing [7], after which the patients' clinical course should be re-evaluated. Evaluating the course of organ dysfunction after 3 days $[9,10]$ or 5 days of ICU care [11] may be a better way to establish confidence for decisions to decide against lifesustaining therapies, if the re-evaluation shows clinical deterioration with no available therapeutic options. However, the use of broader criteria for ICU admission could be associated with an increase in the proportion of deaths occurring after decisions to limit treatment in the ICU. This could lead to an increase in the burden on nurses and physicians and a higher rate of conflicts [12]. If this option is adopted, importance should be attached to the involvement of pulmonologists and/or oncologists directly in the decision and in the communication with the patient's family.

The authors of a survey carried out on behalf of the European Respiratory Society to assess end-of-life practices in patients admitted to European respiratory intermediate care units and high dependency units [5] came to the conclusion that all the 
largest studies, consensus conferences [13] and reviews regarding end-of-life decisions have unfortunately been performed in acute patients admitted to the ICU, and thus may not necessarily apply to chronic respiratory patients and respiratory physicians or associated personnel. In view of the regrettable fact that $\sim 85 \%$ of all lung cancer patients are still dying of cancer in the long term, with every step in the direction of more invasive treatment, the question immediately arises as to the arguments for not employing more invasive treatment, i.e. for establishing criteria for limiting treatment [14]. If lung cancer that can no longer be treated curatively is classified as belonging to the group of advanced chronic lung diseases, we find that among the features that these diseases share are not only a clearly reduced life expectation, but also a permanently impaired quality of life [15]. We need to attach far more weight to these factors when evaluating intensive care than in the past. Irrespective of the usual main goal of intensive therapy, i.e. to eliminate life-threatening conditions or complications, patients with chronic lung disease were found to have an increased rate of depression, communication problems and fear of death and pain after undergoing mechanical ventilation, which calls the success of the treatment into question [16].

In the current practice of lung cancer treatment, it is only very rarely the case that patients, their relatives and significant others are asked for their views at an early stage, before situations such as acute respiratory insufficiency or sepsis and the possible sequelae of intensive care arrive. If this were done, it could help to establish a more reliable basis for future decision-making as the disease progresses for all concerned. Where the scope for medical interventions is limited, both patients' and their relatives' acceptance of the proposed measures are decisively influenced by the participation and communication skills of all involved. A dialogue of this kind should take into account not only current developments in medical technology, but also differences in ethical views and legislative requirements in different countries [5]. It seems absurd that these aspects are not mentioned at all in current reports on the improvements in the results of the intensive care of cancer patients [7].

There is another reason why there are many more layers to the discussion in patients with advanced malignant disease. It has been established that noninvasive ventilation can be employed not only to treat acute respiratory insufficiency, but also as a useful palliative measure in the terminal phase, i.e. without any prospects of or intent to prolong life [17]. However, from an ethical point of view, even though the level of invasiveness is lower, it is still necessary to inform the patient about it just as for intubation, since it is definitely a form of full ventilation [15]. Caution is required especially where the use of noninvasive ventilation is a routine measure as, for example, in a specialised hospital department, since there is a risk that mask ventilation will be used in patients with multiple conditions without consulting them sufficiently beforehand. Decisions on purely palliative interventions in intensive care require social and communication skills similar to those required in the management of diseases of the internal organs. Wherever possible, dialogue should take precedence over making decisions without consulting the patient, which doctors who are accustomed to being action orientated are naturally prone to do. However, if such a dialogue is regularly sought, this is likely to eliminate the risk of making this error in decisions on whether to initiate intensive care more permanently and rapidly than a mere refinement of the classical algorithms of intensive care. Ideally, such ethical and communicative aspects that affect the selection of patients to be transferred to an intensive care unit should also be stipulated as criteria for inclusion in study protocols. The question is, how?

Notwithstanding these uncertainties, what aspects of currently available studies can be summarised with sufficient reliability to really be of help in decision-making? What is most evident from these studies is that attempts to resuscitate incurable lung cancer patients are almost always doomed to fail, i.e. the patient dies within a short time. The prognosis of treatment of acute respiratory insufficiency depends on tumour stage and morphology and thus it differs so widely that it no longer seems possible to formulate an algorithm based on scalable parameters. While there is a substantial difference between patients with nonsmall cell lung carcinoma in stages I and IV (25 versus $88 \%$ 30-day mortality) [2], this figure alone is of little help when it comes to making a decision. None of the studies published to date are suitable, purely in terms of sample size, for drawing conclusions regarding tumour-specific treatment criteria. From the pneumological and oncological standpoints, it is decisive whether patients are at the beginning or the end of their treatment career, but this has never been discussed in the literature. The decision is also not made easier by the fact that it has recently been shown that, even without an objective response, patients may have a favourable prognosis and a good quality of life as long as time to progression can be prolonged [18]. Moreover, a patient's age should not be used as a criterion for deciding for or against intensive care. Research on the evaluation of tumour-specific therapy has already demonstrated that the prognosis is mainly by the stage of the tumour and that it is important not to fall prey to the temptation to make a greater effort to treat particularly young patients than is justified by the stage of their tumours [19]. Comorbidities can be both a reason to limit treatment and an indication for intensive care and can thus only be assessed by taking the whole situation into consideration. Leaving interpretations aside, there are no further recommendations for medical interventions apart from a more or less reliable injunction not to resuscitate [1].

There is, however, one thing that we should definitely do and that is espouse an interdisciplinary approach, both in practice and in our studies, much more than in the past. In addition to the importance of taking the patient's wishes into account, it can only further the decision-making process if representatives of other disciplines, such as psychologists, members of an ethics committee or a hospital chaplain, play a substantial role. To date we are still far from having achieved such a basis for decision making. What usually happens is that a single doctor or team of doctors act on their own. In view of the need for interdisciplinary co-operation in the field of tension between curative and palliative medicine, technology, ethics and legislation, this recommendation would seem even more crucial. Although this may not shift the balance between the indication for intensive care and for limiting treatment for ethical reasons, there is good reason to believe that in the future the debate on the justification for intensive care will 
become more objective and less emotional. This is the strategy that needs to be followed if we aspire to achieve a truly objective evaluation of all relevant prognosis parameters involved, the subjective evaluations of which are currently still causing a rift between doctors and patients.

\section{REFERENCES}

1 Adam AK, Soubani AO. Outcome and prognostic factors of lung cancer patients admitted to the medical intensive care unit. Eur Respir J 2008; 31: 47-53.

2 Reichner CA, Thompson JA, O'Brien S, Kuru T, Anderson TE. Outcome and code status of lung cancer patients admitted to the medical ICU. Chest 2006; 130: 719-723.

3 Lin YC, Tsai YH, Huang CC, et al. Outcome of lung cancer patients with acute respiratory failure requiring mechanical ventilation. Respir Med 2004; 98: 43-51.

4 Garrouste-Orgeas M, Montuclard L, Timsit JF, et al. Predictors of intensive care unit refusal in French intensive care units: a multiple-center study. Crit Care Med 2005; 33 : 750-755.

5 Nava S, Sturani C, Hartl S, et al. End-of-life decisionmaking in respiratory intermediate care units: a European survey. Eur Respir J 2007; 30: 156-164.

6 Soares M, Darmon M, Salluh JI, et al. Prognosis of lung cancer patients with life-threatening complications. Chest 2007; 131: 840-846.

7 Lecuyer L, Chevret S, Thiery G, Darmon M, Schlemmer B, Azoulay E. The ICU trial: a new admission policy for cancer patients requiring mechanical ventilation. Crit Care Med 2007; 35: 808-814.

8 Hilbert G, Gruson D, Vargas F, et al. Noninvasive ventilation in immunosuppressed patients with pulmonary infiltrates, fever, and acute respiratory failure. $N$ Engl J Med 2001; 344: 481-487.
9 Larché J, Azoulay E, Fieux F, et al. Improved survival of critically ill cancer patients with septic shock. Intensive Care Med 2003; 29: 1688-1695.

10 Guiguet M, Blot F, Escudier B, Antoun S, Leclercq B, Nitenberg G. Severity-of-illness scores for neutropenic cancer patients in an intensive care unit: which is the best predictor? Do multiple assessment times improve the predictive value? Crit Care Med 1998; 26: 488-493.

11 Massion PB, Dive AM, Doyen C, et al. Prognosis of hematologic malignancies does not predict intensive care unit mortality. Crit Care Med 2002; 30: 2260-2270.

12 Prendergast TJ. Resolving conflicts surrounding end-of-life care. New Horiz 1997; 5: 62-71.

13 Carlet J, Thijs LG, Antonelli M, et al. Challenges in end-oflife care in the ICU. Statement of the 5th International Consensus Conference in Critical Care: Brussels, Belgium, April 2003. Intensive Care Med 2004; 30: 770-784.

14 Griffin JP, Nelson JE, Koch KA, et al. End-of-life care in patients with lung cancer. Chest 2003; 123: Suppl. 1, 312S-331S.

15 Schönhofer B, Köhler D, Kutzer K. Ethische Betrachtungen zur Beatmungsmedizin unter besonderer Berücksichtigung des Lebensendes. [Ethics of mechanical ventilation in end of life.] Pneumologie 2006; 60: 408-416.

16 Pochard F, Lanore JJ, Bellivier F, et al. Subjective psychological status of severely ill patients discharged from mechanical ventilation. Clin Intensive Care 1995; 6: 57-61.

17 Cuomo A, Delmastro M, Ceriana P, et al. Noninvasive mechanical ventilation as a palliative treatment of acute respiratory failure in patients with end-stage solid cancer. Palliat Med 2004; 18: 602-610.

18 Gollob JA, Bonomi P. Historic evidence and future directions in clinical trial therapy of solid tumors. Oncology (Williston Park) 2006; 20: Suppl. 5, 10-18.

19 Lienert T, Serke M, Schönfeld N, Loddenkemper R. Lung cancer in young females. Eur Respir J 2000; 16: 986-990. 